\section{Prevalência e fatores associados à pressão arterial elevada, seu conhecimento e tratamento em idosos no sul do Brasil}

\author{
Prevalence and factors associated with high blood \\ pressure, awareness, and treatment among elderly \\ in Southern Brazil
}

\author{
Prevalencia y factores asociados a la presión \\ arterial elevada, su conocimiento y tratamiento \\ en ancianos en el sur de Brasil
}

${ }^{1}$ Centro de Ciências da Saúde, Universidade Federal de Santa Catarina, Florianópolis, Brasil.

Correspondência

L. C. Zattar

Centro de Ciências da Saúde, Universidade Federal de Santa Catarina.

Campus Universitário Reitor João David Ferreira Lima,

Florianópolis, SC 88035-200, Brasil.

lucianazattar@hotmail.com

\begin{abstract}
The aim of this study was to describe prevalence, awareness, and treatment of high blood pressure (HBP) and associated factors among the elderly in Florianópolis, Santa Catarina State, Brazil. This cross-sectional population-based study used a complex sampling design. HBP was defined as elevated blood pressure (by direct measurement), use of antihypertensive medication, or prior diagnosis. The association of outcomes with independent variables was assessed by Poisson regression. One-thousand seven hundred and five participants were interviewed. Of these, $84.6 \%$ presented $H B P, 77.5 \%$ were aware of their condition, and $79.1 \%$ were on antihypertensive medication. Prevalence of HBP was associated with age, functional capacity, and body mass index (BMI). Awareness of the condition was associated with age, gender, BMI, self-rated health, and recent medical consultation. Treatment was associated with gender, functional capacity, self-rated health, and recent medical consultation. Although public health policies should include everyone, unequal distribution of HBP in the population should be addressed through targeted preventive, diagnostic, and therapeutic measures.
\end{abstract}

Hypertension; Health of the Elderly; Prevalence
Luciana Carmen Zattar 1

Antonio Fernando Boing 1

Maruí Weber Corseuil Giehl 1

Eleonora d'Orsi 1

\section{Resumo}

O objetivo foi estimar a prevalência e investigar os fatores associados à pressão arterial elevada, seu conhecimento e tratamento em idosos de Florianópolis, Santa Catarina, Brasil. Trata-se de estudo transversal de base populacional e amostra complexa. A pressão arterial elevada foi definida pela medida do nível pressórico ou uso de antihipertensivo, ou diagnóstico prévio. A associação dos desfechos com as variáveis independentes foi verificada pela regressão de Poisson. Foram entrevistados 1.705 idosos. Desses, 84,6\% apresentaram pressão arterial elevada, 77,5\% estavam cientes da doença e 79,1\% a tratavam. A prevalência associou-se à dependência funcional e idade e índice de massa corporal (IMC) elevados. Idosos do sexo feminino, idade e IMC elevados, pior percepção em saúde e consulta médica recente estavam mais cientes da doença. O tratamento associou-se a sexo masculino, dependência funcional, pior percepção em saúde e consulta médica recente. Ainda que políticas públicas em saúde devam ser universais, é preciso considerar a desigual distribuição da pressão arterial elevada na população, direcionando-se ações de prevenção, diagnóstico e garantia de tratamento.

Hipertensão; Saúde do Idoso; Prevalência 


\section{Introdução}

A hipertensão arterial sistêmica (HAS) é o principal fator de risco para doenças cardiovasculares 1 . Estima-se que $54 \%$ dos casos de acidente vascular cerebral e $47 \%$ dos infartos agudos do miocárdio estejam relacionados a elevados níveis pressóricos 2 . Aproximadamente 1 bilhão de pessoas viviam com a hipertensão em 2000, valor que deve aumentar $60 \%$ em pouco mais de duas décadas e chegar a 1,56 bilhão em $2025^{3}$. Além disso, a HAS é responsável por cerca de 7,1 milhões de mortes ao ano no mundo ${ }^{1}$.

No Brasil, as doenças cardiovasculares foram responsáveis por cerca de 300 mil mortes em 2007 4, 40\% das aposentadorias precoces e custo econômico estimado em cerca de 475 milhões de reais apenas em internações hospitalares, que chegam a mais de 1 milhão por ano 5 . Tal impacto está diretamente relacionado aos casos de HAS no país, cuja prevalência média estimada foi de $24 \%$ em 2007 , observando-se valores mais elevados nos grupos de maior idade 6 , atingindo cerca de $50 \%$ dos indivíduos entre 60 e 69 anos e mais de $70 \%$ daqueles acima de 70 anos 4 .

Para o controle e adequado manejo da pressão arterial elevada e de suas consequências é imprescindível a identificação e acompanhamento dos hipertensos pelos serviços de saúde 7, pois tratamentos farmacológicos e não farmacológicos são capazes de melhorar significativamente o prognóstico da doença e a qualidade de vida das pessoas ${ }^{8}$. Um dos determinantes para a busca dessas medidas terapêuticas é o próprio conhecimento da condição de hipertenso 7; no entanto, em muitos casos, esse diagnóstico precoce não ocorre.

Estima-se que cerca de um terço dos hipertensos nunca teve o diagnóstico clínico da doença feito por um profissional de saúde 7 , e muitas vezes tomam ciência da doença apenas após um evento clínico grave decorrente de seu descontrole por vários anos 9 , tais como infarto agudo do miocárdio ou acidentes vasculares encefálicos 10,11. Além disso, grande parte das pessoas que conhecem seu diagnóstico de HAS apresenta baixa aderência ao tratamento ou é tratada com terapêutica incorreta 10,12. Tais situações levam ao baixo controle dos níveis pressóricos mesmo por parte da população ciente de sua condição clínica 10 .

Estudos de base populacional foram realizados para investigar a prevalência e os fatores associados à HAS na população brasileira, no entanto expressiva parte foi direcionada a adultos, adolescentes e crianças 5,9,12,13,14. As pesquisas com idosos restringiram-se à região sudeste do país $5,7,15$, nas quais se verificou prevalência de hipertensão variando entre 51,8\% e 61,5\%. O grau de conhecimento e tratamento da HAS em idosos foi pouco explorado no Brasil 7,16, sendo necessários trabalhos adicionais sobre 0 problema.

O presente estudo tem como objetivo descrever a prevalência, o conhecimento e o tratamento da pressão arterial elevada e seus fatores associados em indivíduos de 60 anos de idade ou mais, na cidade de Florianópolis, Santa Catarina, Brasil.

\section{Métodos}

\section{Considerações éticas}

O projeto foi aprovado pelo comitê de Ética em Pesquisa com Seres Humanos da Universidade Federal de Santa Catarina, protocolo no $352 / 2008$, e foi solicitado aos entrevistados a assinatura do Termo de Consentimento Livre e Esclarecido.

\section{Área e população de estudo}

Trata-se de estudo transversal de base populacional e domiciliar, realizado em 2009-2010, com idosos (pessoas com 60 anos de idade ou mais) não institucionalizados e residentes na zona urbana do Município de Florianópolis. Este trabalho faz parte de um inquérito populacional sobre as condições de saúde da população idosa de Florianópolis denominado EpiFloripa Idoso (http://www.epifloripa.ufsc.br).

Capital do Estado de Santa Catarina, o Município de Florianópolis teve sua população estimada, no ano de 2009 17, em 408.163 habitantes. Desses, 44.460 tinham idades iguais ou superiores a 60 anos. Localizado no leste do estado, o município apresentava esperança de vida ao nascer de 72,8 anos em 200018.

O cálculo do tamanho da amostra considerou os seguintes parâmetros: tamanho da população igual a 44.460, prevalência desconhecida de 50\%, erro amostral igual a 4 pontos percentuais, intervalo de 95\% de confiança (IC95\%) e efeito de delineamento igual a 2. Foram acrescidos, ainda, $20 \%$ para perdas previstas e $15 \%$ para controle de fatores de confusão em estudos de associação, totalizando uma amostra desejável de, no mínimo, 1.599 pessoas.

A amostragem foi realizada por conglomerados em dois estágios. Com base nos dados do Instituto Brasileiro de Geografia e Estatística (IBGE), os 420 setores censitários urbanos do município foram ordenados pela média da renda mensal dos chefes de família domiciliados no setor e estratificados em decis. Em seguida, sortearam-se sis- 
tematicamente 80 setores (oito em cada decil de renda). As unidades do segundo estágio foram os domicílios. Como o registro dos domicílios em cada setor datava do ano 2000 (último censo conduzido antes do presente estudo), foi realizada a atualização do número de domicílios particulares habitados em cada unidade. Verificou-se que esse número variou de 61 a 725 e, para diminuir o coeficiente de variação no número de domicílios das unidades amostrais, foi realizada a fusão e a divisão de setores, respeitando-se o decil de renda correspondente e a proximidade geográfica. Assim, o coeficiente de variação inicial passou de $52,7 \%$ ( $n=80$ setores) para $35,2 \%$ ( $n=83$ setores). A seguir, sortearam-se os domicílios de maneira sistemática, sendo que todos os idosos residentes nos domicílios sorteados foram convidados a participar do estudo.

Consideraram-se perdas as entrevistas não realizadas após quatro tentativas (inclusive no período noturno e no final de semana) e recusas quando as pessoas optaram em não responder ao questionário.

\section{Coleta de dados}

A coleta de dados foi realizada por entrevistadoras treinadas, com ensino médio completo e disponibilidade integral para a realização do trabalho de campo. Foi aplicado um questionário estruturado na forma de entrevistas face a face, utilizando-se personal digital assistant (PDA) para registro das informações. Anteriormente à coleta de dados, foi realizado um pré-teste do questionário com 30 idosos e um estudo piloto com 99 idosos em setores não amostrados para a pesquisa. Semanalmente foi verificada a consistência dos dados e realizado o controle de qualidade usando-se a aplicação por telefone de um questionário reduzido em $10 \%$ das entrevistas selecionadas aleatoriamente.

Para o trabalho, considerou-se com pressão arterial elevada o idoso que apresentou pressão arterial sistólica $\geq 140 \mathrm{mmHg}$ (PAS $\geq 140 \mathrm{mmHg}$ ) e/ou diastólica $\geq 90 \mathrm{mmHg}$ (PAD $\geq 90 \mathrm{mmHg})^{4}$, ou que fazia uso de medicação anti-hipertensiva ou que reportou diagnóstico de hipertensão por profissional de saúde, parâmetros empregados em outros estudos 8,19.

A mensuração da pressão arterial foi realizada por meio de duas medidas em cada braço, sendo uma no início da entrevista e outra aproximadamente 40 minutos após o seu início. A pressão foi aferida com aparelhos eletrônicos de leitura digital do modelo Z-40 da marca Techline (Techline, Taiwan, China) devidamente calibrados para a pesquisa. Para análise no presente estudo foram calculadas as médias para cada braço e o nível pressórico final foi considerado como a média do braço de maior valor 4 .

Em todos os indivíduos foi verificado o uso de medicamentos fazendo-se a pergunta: "Nos últimos 30 dias, o(a) sr.(a) usou algum remédio?". Caso o entrevistado respondesse positivamente, procedia-se os registros dos medicamentos consumidos nesse período, identificando-se sua substância ativa e indicação, e classificandose o insumo segundo a Anatomical Therapeutic Chemical (ATC), criada pela WHO Collaborating Centre for Drug Statistics Methodology 20. Assim, com o uso de medicação anti-hipertensiva foi considerado que o idoso hipertenso estava em tratamento.

Também foi verificada a existência de diagnóstico de pressão arterial elevada por profissional de saúde. A informação foi autorreferida usando-se a pergunta: "Algum médico ou profissional de saúde já disse que o(a) senhor(a) tem pressão alta ou hipertensão arterial?" 8. Caso respondesse sim à pergunta, o entrevistado era considerado ciente de sua doença.

As variáveis exploratórias demográficas e socioeconômicas consideradas foram sexo, idade (60-69 anos, 70-79 e 80 ou mais), cor da pele autorreferida (branca, parda ou preta; amarelos $(n=12)$ e indígenas $(n=16)$ foram excluídos por representarem pequena amostra), escolaridade ( $\leq 4$ anos de estudos, 5-8, 9-11 e $\geq 12$ ) e renda familiar per capita estratificada em quartis (1o quartil: $\leq \mathrm{R} \$ 327,50$, 2o: entre R\$ 327,50 e R\$ $700,00,3$ o: entre $\mathrm{R} \$ 700,00$ e $\mathrm{R} \$ 1.500,00$, e $4 \underline{\text { o }}$ quartil: > R\$ 1.500,00).

Também foram investigados o tabagismo (fumante atual, ex-fumante ou nunca fumou), o consumo de bebida alcoólica (nunca, moderado, alto ou de risco; foi considerado alto ou de risco o relato de consumo de 3 ou mais doses em um dia típico, ou relato de 6 ou mais doses de uma só vez (uso excessivo episódico ou binge drinking), informação verificada por meio das três primeiras perguntas do questionário AUDIT (The Alcohol Use Disorders Identification Test) 21, e a atividade física de lazer e deslocamento (verificada pelo Questionário Internacional de Atividade Física - IPAQ, versão longa), sendo os indivíduos classificados em fisicamente ativos ( $\geq 150$ minutos de atividades físicas/semana) e insuficientemente ativos $(<150$ minutos de atividades físicas/semana) 22 .

As condições de saúde analisadas foram: (1) dependência funcional, verificada por meio da aplicação da escala de atividades da vida diária (AVD) 23 , sendo classificada em ausência de dependência, dependência leve e dependência moderada/grave; (2) índice de massa corporal, classificado em baixo peso (IMC $<22,0 \mathrm{~kg} / \mathrm{m}^{2}$ ), 
peso adequado $\left(22,0 \leq \mathrm{IMC} \leq 27,0 \mathrm{~kg} / \mathrm{m}^{2}\right)$ e sobrepeso/obesidade (IMC $>27,0 \mathrm{~kg} / \mathrm{m}^{2}$ ) 24; e (3) autopercepção de saúde (muito boa/boa, regular e ruim/muito ruim). Por fim, foi verificado o uso do serviço de saúde por meio da pergunta "Nos últimos 3 meses o(a) sr.(a) consultou com médico?”, e posse de plano de saúde, informação obtida pela pergunta " $O(a)$ sr.(a) tem plano de saúde particular, de empresa ou órgão público?”.

\section{Análise dos dados}

Foram calculadas as prevalências de pressão arterial elevada, seu conhecimento e tratamento segundo as categorias das variáveis exploratórias. Em seguida, testaram-se as associações entre os desfechos e as variáveis independentes em análise bivariada e multivariada com Regressão de Poisson. Foram estimadas as razões de prevalência (RP) brutas e ajustadas com seus respectivos intervalos de $95 \%$ de confiança (IC95\%) 25. Com base nos resultados da análise bivariada foram incluídas na análise multivariada todas as variáveis que apresentaram $\mathrm{p}<$ 0,20 , permanecendo no modelo final aquelas com $\mathrm{p}<0,05 \mathrm{e} /$ ou que ajustassem o modelo 25 . A modelagem foi realizada com a entrada das variáveis passo a passo, ordenadas do menor para o maior valor de $\mathrm{p}$.

A análise dos dados foi conduzida no programa estatístico Stata 11.0 (Stata Corp., College Station, Estados Unidos).Todas as análises foram realizadas considerando o efeito do desenho amostral por conglomerados e incorporando-se os pesos amostrais usando-se o comando svy.

\section{Resultados}

Nos domicílios sorteados foram identificados e convidados a participar da pesquisa 1.911 idosos, sendo efetivamente entrevistados 1.705 (taxa de resposta de $89,2 \%$ ). A maior parte da população do trabalho foi do sexo feminino, a média de idade foi de 70,7 anos e $87 \%$ referiram ter a cor da pele branca. Expressiva proporção dos idosos tinha de 0-4 anos de estudos e a renda per capita média foi de $\mathrm{R} \$ 1.347,15$ (desvio-padrão = $\mathrm{R} \$ 2.610,17)$. A maioria dos entrevistados nunca fumou, não reportou uso excessivo de bebida alcoólica, foi classificada como insuficientemente ativa e relatou alguma dependência para realização de atividades da vida diária (Tabela 1).

A prevalência de idosos com pressão arterial elevada foi de 84,6\% (IC95\%: 82,9-86,3). Observou-se maior prevalência entre aqueles com idades de 70-79 anos (90,4\%), com menor escolaridade $(87,5 \%)$, que nunca fumaram $(86 \%)$ ou não reportaram consumo excessivo de bebida alcoólica (86,2\%). A pressão arterial elevada foi mais prevalente entre os idosos com sobrepeso ou obesidade $(90,5 \%)$, percepção da saúde ruim/ muito ruim $(90,3 \%)$, que havia consultado um médico recentemente $(85,9 \%)$ e possuía dependência funcional de moderada a grave $(88,7 \%)$ (Tabela 1).

O critério que isoladamente identificou mais indivíduos hipertensos foi a medida elevada do nível pressórico no momento da entrevista (22,8\%). Dos idosos identificados com pressão arterial elevada, mais de 3/4 sabiam ter a doença. Porém, desses idosos conscientes de sua situação, aproximadamente 1/5 não estava em tratamento (Tabela 2).

A Tabela 3 apresenta os fatores associados à pressão arterial elevada. Observou-se na análise univariada associação do desfecho com idade, tabagismo, dependência funcional leve, dependência de moderada a grave, índice de massa corporal e autopercepção de saúde. Já na análise multivariada, verificou-se que a prevalência de pressão arterial elevada nos idosos de 70-79 anos de idade foi $11 \%$ maior que naqueles de 60-69 anos (RP = 1,11; IC95\%: 1,05-1,18), e que o desfecho foi mais comum entre as pessoas com dependência funcional de moderada a grave $(\mathrm{RP}=1,09$; IC95\%: 1,01-1,18) e entre as pessoas com sobrepeso/obesidade $(\mathrm{RP}=1,10$; IC95\%: 1,04-1,15).

Os fatores associados ao conhecimento da condição de pressão arterial elevada são apresentados na Tabela 4. Na análise univariada, as únicas variáveis que não se demonstraram associadas foram cor da pele e plano de saúde. Na análise multivariada, constatou-se que a prevalência do conhecimento da doença foi $21 \%$ maior entre as mulheres ( $\mathrm{RP}=1,21$; IC95\%: 1,13-1,30) e $25 \%$ maior entre os idosos com mais de 80 anos quando comparados com aqueles de 60 a 69 anos (RP = 1,25; IC95\%: 1,15-1,36). O desfecho também foi mais frequente entre pessoas com sobrepeso e obesidade (RP = 1,11; IC95\%: 1,02-1,20), pior percepção de saúde $(R P=1,23$; IC95\%: 1,12-1,35) e que tiveram consulta médica recente $(\mathrm{RP}=1,22$; IC95\%: 1,11-1,33).

Na Tabela 5, são apresentados os fatores associados ao tratamento da pressão arterial elevada. Verificou-se associação do desfecho na análise bruta com sexo, dependência funcional, pior autopercepção da saúde e consulta médica recente. Na análise multivariada, a prevalência de ter um tratamento instituído foi $13 \%$ maior entre idosos com dependência funcional de moderada a grave $(R P=1,13$; IC95\%: 1,01-1,27) e entre aqueles com percepção de saúde ruim/muito ruim ( $\mathrm{RP}=$ 1,13; IC95\%: 1,04-1,22), quando comparados, res- 
Prevalência de pressão arterial elevada, seu conhecimento e tratamento em idosos segundo variáveis sociodemográficas, hábitos de vida, condições de saúde e uso de serviços de saúde. Florianópolis, Santa Catarina, Brasil, 2009-2010.

\begin{tabular}{|c|c|c|c|c|c|c|c|}
\hline \multirow[t]{2}{*}{ Variáveis } & \multirow[t]{2}{*}{ n (\%) } & \multicolumn{2}{|c|}{ Pressão arterial elevada } & \multicolumn{2}{|c|}{ Conhecimento } & \multicolumn{2}{|c|}{ Tratamento } \\
\hline & & $\%$ & IC95\% & $\%$ & IC95\% & $\%$ & IC95\% \\
\hline \multicolumn{8}{|l|}{ Sexo } \\
\hline Masculino & $616(36,1)$ & 85,6 & $82,8-88,3$ & 68,31 & $64,33-72,30$ & 84,87 & $81,14-88,61$ \\
\hline Feminino & $1.089(63,9)$ & 84,1 & $81,9-86,3$ & 82,86 & $80,42-85,31$ & 76,38 & $73,32-79,43$ \\
\hline \multicolumn{8}{|l|}{ Faixa etária (anos) } \\
\hline $60-69$ & $854(50,1)$ & 79,9 & $77,2-82,6$ & 70,82 & $67,40-74,24$ & 76,72 & $72,92-80,51$ \\
\hline $70-79$ & $612(35,9)$ & 90,4 & $88,0-92,7$ & 82,46 & $79,28-85,64$ & 80,18 & $76,48-83,88$ \\
\hline 80 ou mais & $239(14,0)$ & 87,0 & $82,7-91,3$ & 86,46 & $81,86-91,22$ & 83,14 & $77,49-88,79$ \\
\hline \multicolumn{8}{|l|}{ Cor da pele (referida) } \\
\hline Branca & $1.444(87,0)$ & 84,6 & $82,8-86,5$ & 76,92 & $74,56-79,29$ & 79,44 & $76,84-82,04$ \\
\hline Parda e preta & $215(13,0)$ & 85,6 & $80,8-90,3$ & 80,98 & $75,25-86,70$ & 78,23 & $71,48-84,98$ \\
\hline \multicolumn{8}{|l|}{ Escolaridade (anos) } \\
\hline 12 ou mais & $394(23,2)$ & 82,2 & $78,4-86,0$ & 69,75 & $64,73-74,78$ & 78,73 & $73,30-84,17$ \\
\hline $9-11$ & $234(13,8)$ & 81,6 & $76,6-86,6$ & 73,30 & $66,97-79,63$ & 79,29 & $72,49-86,08$ \\
\hline $5-8$ & $321(19,0)$ & 83,2 & $79,1-87,3$ & 78,65 & $73,70-83,60$ & 81,73 & $76,65-85,68$ \\
\hline $0-4$ & $745(44,0)$ & 87,5 & $85,1-89,9$ & 82,06 & $79,10-85,01$ & 78,75 & $75,24-82,25$ \\
\hline \multicolumn{8}{|l|}{ Renda familiar per capita } \\
\hline 4o quartil (maior) & $418(24,5)$ & 82,8 & $79,1-86,4$ & 73,41 & $68,73-78,09$ & 77,87 & $72,72-83,02$ \\
\hline 3o quartil & $425(24,9)$ & 85,4 & $82,0-88,8$ & 77,41 & $73,09-81,73$ & 78,18 & $73,27-83,09$ \\
\hline 2o quartil & $435(25,5)$ & 86,4 & $83,2-89,7$ & 79,79 & $75,71-83,86$ & 81,16 & $76,65-85,68$ \\
\hline 1ㅇ quartil & $427(25,1)$ & 83,8 & $80,3-87,3$ & 79,33 & $75,11-83,54$ & 79,08 & $74,30-83,85$ \\
\hline \multicolumn{8}{|l|}{ Tabagismo } \\
\hline Nunca fumou & $1.039(61,0)$ & 86,0 & $83,9-88,2$ & 80,43 & $77,82-83,03$ & 77,40 & $74,31-80,49$ \\
\hline Fumou e parou & $523(30,7)$ & 85,7 & $82,6-88,7$ & 74,78 & $70,74-78,81$ & 83,03 & $78,96-87,10$ \\
\hline Fumante atual & $141(8,3)$ & 70,9 & $63,3-78,5$ & 64,00 & $54,45-73,57$ & 79,13 & $67,72-88,53$ \\
\hline \multicolumn{8}{|l|}{ Consumo de bebida alcoólica } \\
\hline Nunca & $1.108(65,0)$ & 86,2 & $84,2-88,2$ & 81,78 & $79,33-84,23$ & 80,05 & $77,22-82,89$ \\
\hline Moderado & $316(18,5)$ & 83,2 & $79,1-87,4$ & 75,29 & $70,04-80,53$ & 77,95 & $72,08-83,82$ \\
\hline Alto & $281(16,5)$ & 80,1 & $75,4-84,8$ & 62,22 & $55,84-68,61$ & 75,71 & $68,52-82,91$ \\
\hline \multicolumn{8}{|l|}{ Atividade física } \\
\hline Fisicamente ativos & $494(29,0)$ & 84,6 & $81,4-87,8$ & 70,33 & $65,94-74,73$ & 76,37 & $71,47-81,27$ \\
\hline Insuficientemente ativos & $1.211(71,0)$ & 84,6 & $82,6-86,7$ & 80,49 & $78,06-82,92$ & 80,12 & $77,37-82,88$ \\
\hline \multicolumn{8}{|l|}{ Dependência funcional } \\
\hline Ausência de dependência & $458(26,9)$ & 79,0 & $75,3-82,8$ & 68,78 & $63,99-73,58$ & 73,39 & $67,85-78,93$ \\
\hline Dependência leve & $709(41,6)$ & 85,2 & $82,6-87,8$ & 73,68 & $70,15-77,20$ & 79,23 & $75,52-83,73$ \\
\hline Dependência moderada/grave & $538(31,5)$ & 88,7 & $86,0-91,3$ & 89,10 & $86,29-91,91$ & 82,48 & $78,79-86.17$ \\
\hline \multicolumn{8}{|l|}{ IMC (peso/altura2) } \\
\hline Peso adequado & $641(38,9)$ & 81,0 & $77,9-84,0$ & 71,87 & $67,99-75,75$ & 79,62 & $75,52-83,73$ \\
\hline Baixo peso & $140(8,5)$ & 69,3 & $61,5-77,0$ & 71,13 & $61,95-80,31$ & 69,12 & $57,85-80,38$ \\
\hline Sobrepeso/Obesidade & $865(52,6)$ & 90,5 & $88,6-92,5$ & 81,35 & $78,62-84,09$ & 79,24 & $76,07-82,41$ \\
\hline \multicolumn{8}{|l|}{ Percepção em saúde } \\
\hline Muito boa/Boa & $860(51,2)$ & 82,0 & $79,4-84,6$ & 68,65 & $65,22-72,09$ & 73,91 & $69,98-77,84$ \\
\hline Regular & $646(38,4)$ & 87,2 & $84,6-89,7$ & 83,66 & $80,60-86,72$ & 82,62 & $79,16-86,07$ \\
\hline Ruim/Muito ruim & $175(10,4)$ & 90,3 & $85,9-89,7$ & 93,04 & $89,03-97,05$ & 84,72 & $78,78-90,67$ \\
\hline
\end{tabular}

(continua) 
Tabela 1 (continuação)

\begin{tabular}{|c|c|c|c|c|c|c|c|}
\hline \multirow[t]{2}{*}{ Variáveis } & \multirow[t]{2}{*}{ n (\%) } & \multicolumn{2}{|c|}{ Pressão arterial elevada } & \multicolumn{2}{|c|}{ Conhecimento } & \multicolumn{2}{|c|}{ Tratamento } \\
\hline & & $\%$ & IC95\% & $\%$ & IC95\% & $\%$ & IC95\% \\
\hline \multicolumn{8}{|c|}{ Plano de saúde } \\
\hline Sim & $1.087(63,8)$ & 84,5 & $82,3-86,6$ & 76,69 & $73,95-79,43$ & 79,34 & $76,33-82,35$ \\
\hline Não & $618(36,2)$ & 85,0 & $82,1-87,8$ & 79,05 & $75,56-82,54$ & 78,77 & $74,77-82,77$ \\
\hline \multicolumn{8}{|c|}{ Consulta médica recente } \\
\hline Sim & $1.224(71,8)$ & 85,9 & $83,9-87,8$ & 82,02 & $79,69-84,34$ & 81,53 & $78,92-84,14$ \\
\hline Não & $481(28,2)$ & 81,5 & $78,0-85,0$ & 65,56 & $60,84-70,29$ & 71,03 & $65,39-76,67$ \\
\hline
\end{tabular}

IC95\%: intervalo de 95\% de confiança.

Tabela 2

Distribuição de idosos com pressão arterial elevada, de acordo com critérios diagnósticos e tratamento. Florianópolis, Santa Catarina, Brasil, 2009-2010.

$\%$

\begin{tabular}{|c|c|}
\hline \multicolumn{2}{|l|}{ População total } \\
\hline Idosos com pressão arterial elevada * & 84,6 \\
\hline \multicolumn{2}{|l|}{ Pressão arterial elevada de acordo com diferentes critérios } \\
\hline Somente diagnóstico positivo & 4,9 \\
\hline Somente medicação anti-hipertensiva & 4,8 \\
\hline Somente medida de PAS $\geq 140 \mathrm{mmHg}$ e $\mathrm{PAD} \geq 90 \mathrm{mmHg}$ & 22,8 \\
\hline Diagnóstico e medicação anti-hipertensiva & 15,2 \\
\hline Diagnóstico e medida de PAS $\geq 140 \mathrm{mmHg}$ e PAD $\geq 90 \mathrm{mmHg}$ & 11,6 \\
\hline Medicação anti-hipertensiva e medida de PAS $\geq 140 \mathrm{mmHg}$ e PAD $\geq 90 \mathrm{mmHg}$ & 3,3 \\
\hline Diagnóstico, medicação e medida de PAS $\geq 140 \mathrm{mmHg}$ e PAD $\geq 90 \mathrm{mmHg}$ & 37,4 \\
\hline \multicolumn{2}{|l|}{ Idosos com pressão arterial elevada * } \\
\hline Consciente de ter a doença & 77,5 \\
\hline Desconhecem ter a doença & 22,5 \\
\hline \multicolumn{2}{|l|}{ Idosos conscientes de sua pressão arterial elevada } \\
\hline Estão em tratamento & 79,1 \\
\hline Não estão em tratamento & 20,9 \\
\hline
\end{tabular}

pectivamente, com aqueles sem dependência funcional e boa percepção de saúde. Também a realização de consulta médica nos últimos 3 meses ( $R P=1,15$; IC95\%: 1,05-1,26) e não ser do sexo feminino ( $R P=0,89$; IC95\%: 0,83-0,94) foram significativamente associados ao tratamento antihipertensivo.

\section{Discussão}

$\mathrm{Na}$ população estudada verificou-se alta prevalência de pressão arterial elevada, expressi- va proporção de idosos que desconheciam esta condição e que uma em cada cinco pessoas com pressão elevada não estava em tratamento. A prevalência de pressão arterial elevada associou-se à dependência funcional, idade e IMC elevados. O conhecimento associou-se a sexo feminino, idade e IMC elevados, pior percepção da saúde e consulta médica recente. Já o tratamento associou-se a sexo, dependência funcional, percepção ruim da saúde e consulta médica recente.

A prevalência de pressão arterial elevada entre idosos identificada no presente trabalho variou 
Análises univariada e multivariada dos fatores associados à pressão arterial elevada dos idosos. Florianópolis, Santa Catarina, Brasil, 2009-2010.

\begin{tabular}{|c|c|c|c|c|}
\hline \multirow[t]{2}{*}{ Variáveis } & \multicolumn{2}{|c|}{ Análise univariada } & \multicolumn{2}{|c|}{ Análise multivariada } \\
\hline & RP (IC95\%) & Valor de $p$ * & RP (IC95\%) & Valor de $p$ * \\
\hline Sexo & & 0,766 & & \\
\hline Masculino & 1,00 & & ** & \\
\hline Feminino & $0,99(0,95-1,04)$ & & $\star \star$ & \\
\hline Faixa etária (anos) & & 0,044 & & 0,045 \\
\hline $60-69$ & 1,00 & & 1,00 & \\
\hline $70-79$ & $1,11(1,05-1,18)$ & & $1,11(1,05-1,18)$ & \\
\hline 80 ou mais & $1,04(0,94-1,14)$ & & $1,04(0,94-1,14)$ & \\
\hline Cor da pele (referida) & & 0,683 & $\star \star *$ & \\
\hline Branca & 1,00 & & $\star \star$ & \\
\hline Parda e preta & $1,02(1,00-1,04)$ & & $\star \star$ & \\
\hline Escolaridade (anos) & & 0,078 & & 0,148 \\
\hline 12 ou mais & 1,00 & & 1,00 & \\
\hline $9-11$ & $0,94(0,83-1,07)$ & & $0,93(0,82-1,06)$ & \\
\hline $5-8$ & $0,99(0,92-1,08)$ & & $0,99(0,91-1,07)$ & \\
\hline $0-4$ & $1,04(0,98-1,11)$ & & $1,03(0,96-1,10)$ & \\
\hline Renda familiar per capita & & 0,992 & & \\
\hline 4ㅇ quartil (maior) & 1,00 & & ** & \\
\hline 3ㅇ quartil & $1,03(0,98-1,08)$ & & $\star \star$ & \\
\hline 2o quartil & $1,03(0,97-1,10)$ & & $\star \star$ & \\
\hline 1ㅇquartil & $1,00(0,92-1,07)$ & & ** & \\
\hline Tabagismo & & 0,031 & & 0,056 \\
\hline Nunca fumou & 1,00 & & 1,00 & \\
\hline Fumou e parou & $0,99(0,94-1,04)$ & & $0,98(0,93-1,04)$ & \\
\hline Fumante atual & $0,87(0,77-0,98)$ & & $0,88(0,77-1,00)$ & \\
\hline Consumo de bebida alcoólica & & 0,076 & & 0,314 \\
\hline Nunca & 1,00 & & 1,00 & \\
\hline Moderado & $0,98(0,92-1,04)$ & & $0,99(0,93-1,05)$ & \\
\hline Alto & $0,94(0,86-1,02)$ & & $0,95(0,86-1,06)$ & \\
\hline Atividade física de lazer/deslocamento & & 0,632 & & \\
\hline Fisicamente ativos & 1,00 & & ** & \\
\hline Insuficientemente ativos & $0,99(0,94-1,04)$ & & ** & \\
\hline Capacidade funcional & & $<0,001$ & & 0,015 \\
\hline Ausência de dependência & 1,00 & & 1,00 & \\
\hline Dependência leve & $1,08(1,02-1,15)$ & & $1,06(1,00-1,13)$ & \\
\hline Dependência moderada/grave & $1,14(1,07-1,21)$ & & $1,09(1,01-1,18)$ & \\
\hline IMC (peso/altura2) & & $<0,001$ & & $<0,001$ \\
\hline Peso adequado & 1,00 & & 1,00 & \\
\hline Baixo peso & $0,85(0,75-0,96)$ & & $0,83(0,73-0,94)$ & \\
\hline Sobrepeso/Obesidade & $1,11(1,05-1,17)$ & & $1,10(1,04-1,15)$ & \\
\hline Percepção em saúde & & 0,029 & & 0,587 \\
\hline Muito boa/Boa & 1,00 & & 1,00 & \\
\hline Regular & $1,07(1,02-1,13)$ & & $1,03(0,97-1,09)$ & \\
\hline Ruim/Muito ruim & $1,07(0,97-1,18)$ & & $1,02(0,91-1,14)$ & \\
\hline
\end{tabular}

(continua) 
Tabela 3 (continuação)

\begin{tabular}{|c|c|c|c|c|}
\hline \multirow[t]{2}{*}{ Variáveis } & \multicolumn{2}{|c|}{ Análise univariada } & \multicolumn{2}{|c|}{ Análise multivariada } \\
\hline & RP (IC95\%) & Valor de $p$ * & RP (IC95\%) & Valor de $p$ * \\
\hline Plano de saúde & & 0,665 & & \\
\hline Sim & 1,00 & & $\star \star$ & \\
\hline Não & $0,99(0,93-1,05)$ & & ** & \\
\hline Consulta médica recente & & 0,045 & & 0,160 \\
\hline Não & 1,00 & & 1,00 & \\
\hline Sim & $0,94(0,88-1,00)$ & & $0,95(0,90-1,10)$ & \\
\hline
\end{tabular}

IC95\%: intervalo de 95\% de confiança; IMC: índice de massa corporal; RP: razão de prevalência.

* Valor de p corresponde ao teste de Wald da Regressão de Poisson;

** Variável excluída do modelo múltiplo por apresentar valor de $p>0,05$.

Tabela 4

Análises univariada e multivariada dos fatores associados ao conhecimento da condição de hipertenso pelos idosos. Florianópolis, Santa Catarina, Brasil, 2009-2010.

\begin{tabular}{|c|c|c|c|c|}
\hline \multirow[t]{2}{*}{ Variáveis } & \multicolumn{2}{|c|}{ Análise univariada } & \multicolumn{2}{|c|}{ Análise multivariada } \\
\hline & RP (IC95\%) & Valor de $p$ * & RP (IC95\%) & Valor de $p$ * \\
\hline Sexo & & $<0,001$ & & $<0,001$ \\
\hline Masculino & 1,00 & & 1,00 & \\
\hline Feminino & $1,24(1,15-1,33)$ & & $1,21(1,13-1,30)$ & \\
\hline Faixa etária (anos) & & $<0,001$ & & $<0,001$ \\
\hline $60-69$ & 1,00 & & 1,00 & \\
\hline 70-79 & $1,15(1,06-1,25)$ & & $1,13(1,05-1,22)$ & \\
\hline 80 ou mais & $1,27(1,16-1,38)$ & & $1,25(1,15-1,36)$ & \\
\hline Cor da pele (referida) & & 0,130 & & 0,055 \\
\hline Branca & 1,00 & & 1,00 & \\
\hline Parda e preta & $1,07(0,98-1,16)$ & & $1,08(0,99-1,16)$ & \\
\hline Escolaridade (anos) & & $<0,001$ & & 0,014 \\
\hline 12 ou mais & 1,00 & & 1,00 & \\
\hline $9-11$ & $1,04(0,98-1,19)$ & & $0,97(0,86-1,11)$ & \\
\hline $5-8$ & $1,17(1,04-1,31)$ & & $1,07(0,95-1,22)$ & \\
\hline $0-4$ & $1,20(1,09-1,31)$ & & $1,10(0,99-1,22)$ & \\
\hline Renda familiar per capita & & 0,049 & & 0,857 \\
\hline 4o quartil (maior) & 1,00 & & 1,00 & \\
\hline 3ㅇ quartil & $1,10(0,98-1,23)$ & & $1,08(0,96-1,21)$ & \\
\hline 2o quartil & $1,14(1,02-1,28)$ & & $1,08(0,95-1,22)$ & \\
\hline 1ㅇquartil & $1,09(0,98-1,22)$ & & $1,02(0,90-1,15)$ & \\
\hline Tabagismo & & 0,001 & & 0,478 \\
\hline Nunca fumou & 1,00 & & 1,00 & \\
\hline Fumou e parou & $1,07(0,91-1,25)$ & & $1,03(0,88-1,21)$ & \\
\hline Fumante atual & $1,20(1,04-1,38)$ & & $1,05(0,90-1,23)$ & \\
\hline Consumo de bebida alcoólica & & $<0,001$ & & 0,365 \\
\hline Nunca & 1,00 & & 1,00 & \\
\hline Moderado & $1,20(1,07-1,34)$ & & $1,08(0,97-1,22)$ & \\
\hline Alto & $1,29(1,14-1,45)$ & & $1,09(0,94-1,25)$ & \\
\hline
\end{tabular}

(continua) 


\begin{tabular}{|c|c|c|c|c|}
\hline \multirow[t]{2}{*}{ Variáveis } & \multicolumn{2}{|c|}{ Análise univariada } & \multicolumn{2}{|c|}{ Análise multivariada } \\
\hline & RP (IC95\%) & Valor de $p$ * & RP (IC95\%) & Valor de $p$ * \\
\hline Atividade física de lazer/deslocamento & & 0,012 & & 0,108 \\
\hline Fisicamente ativos & 1,00 & & 1,00 & \\
\hline Insuficientemente ativos & $1,13(1,02-1,25)$ & & $1,07(0,98-1,17)$ & \\
\hline Dependência funcional & & $<0,001$ & & 0,065 \\
\hline Ausência de dependência & 1,00 & & 1,00 & \\
\hline Dependência leve & $1,04(0,88-1,24)$ & & $0,99(0,84-1,16)$ & \\
\hline Dependência moderada/grave & $1,31(1,12-1,53)$ & & $1,14(0,97-1,34)$ & \\
\hline IMC (peso/altura²) & & 0,002 & & 0,012 \\
\hline Peso adequado & 1,00 & & 1,00 & \\
\hline Baixo peso & $1,02(0,86-1,21)$ & & $0,99(0,85-1,15)$ & \\
\hline Sobrepeso/Obesidade & $1,13(1,05-1,23)$ & & $1,11(1,02-1,20)$ & \\
\hline Percepção em saúde & & $<0,001$ & & $<0,001$ \\
\hline Muito boa/Boa & 1,00 & & 1,00 & \\
\hline Regular & $1,26(1,18-1,36)$ & & $1,16(1,08-1,25)$ & \\
\hline Ruim/Muito ruim & $1,40(1,29-1,53)$ & & $1,23(1,12-1,35)$ & \\
\hline Plano de saúde & & 0,352 & & $\star \star$ \\
\hline $\operatorname{Sim}$ & 1,00 & & ** & \\
\hline Não & $1,04(0,96-1,11)$ & & $\star \star$ & \\
\hline Consulta médica recente & & $<0,001$ & & $<0,001$ \\
\hline Não & 1,00 & & 1,00 & \\
\hline Sim & $1,24(1,13-1,36)$ & & $1,22(1,11-1,33)$ & \\
\hline
\end{tabular}

* Valor de p corresponde ao teste de Wald da Regressão de Poisson.

** Variável excluída do modelo múltiplo por apresentar valor de p > 0,05.

IC95\%: intervalo de 95\% de confiança; IMC: índice de massa corporal; RP: razão de prevalência.

frente a outros estudos; parte destas variações observadas podem ser decorrentes de diferenças metodológicas na determinação do desfecho 26,27, e talvez possa estar superestimada, uma vez que o nível pressórico final foi considerado como a média do braço de maior valor, condizente com a $V I$ Diretrizes Brasileiras de Hipertensão, que afirmam que: "as medidas devem ser obtidas em ambos os braços e, em caso de diferença, deve-se utilizar como referência sempre o braço com o maior valor para as medidas subsequentes" 4 (p. 6).

Quando comparamos os nossos valores aos de Méndez-Chacón et al. 8 na Costa Rica, que também utilizaram os três critérios para compor o desfecho, a prevalência de pressão arterial elevada é substancialmente mais alta $(81,3 \%$ versus $64,5 \%)$. Quando considerado somente o autorrelato de pressão arterial elevada, o valor do presente estudo $(59,1 \%)$ foi semelhante ao reportado em Campinas (51,8\%) e em São Paulo (51,8 a 62\%) 5,15,28. Já quando consideram-se a medida do nível pressórico e/ou o uso de anti-hipertensivo para definir pressão arterial elevada, a prevalência do presente estudo também foi semelhante às taxas europeias, de $89 \%$ na Grécia 29 e $81 \%$ na Inglater- ra ${ }^{30}$, mas superior à encontrada em trabalho brasileiro $(61,5 \%) 7$.

É expressivo que mais de oito em cada dez idosos foram identificados com pressão arterial elevada. A literatura aponta que altas prevalências poderiam estar associadas a fatores genéticos de predisposição à doença, maior exposição a fatores de risco ou diferenças em acesso e uso de serviços de saúde 1,4,27. Não existem ainda variantes genéticas que possam ser utilizadas para predizer o risco individual de HAS, e, tampouco, temos conhecimento de explicações disponíveis na literatura, no campo biológico, sobre as distintas prevalências observadas entre os idosos de Florianópolis e de outras regiões brasileiras. Além disso, dados do VIGITEL Brasil 2011 (Vigilância de Fatores de Risco e Proteção para Doenças Crônicas por Inquérito Telefônico) indicam que a população de Florianópolis apresenta, de maneira geral, melhores hábitos de saúde e estilo de vida em comparação com outras capitais do Brasil 31. Assim, uma hipótese explicativa para a alta prevalência de pressão arterial elevada observada no presente estudo é o bom acesso aos serviços de saúde, ilustrado 
Análises univariada e multivariada dos fatores associados ao tratamento da pressão arterial elevada dos idosos com conhecimento da sua condição de hipertenso. Florianópolis, Santa Catarina, Brasil, 2009-2010.

\begin{tabular}{|c|c|c|c|c|}
\hline \multirow[t]{2}{*}{ Variáveis } & \multicolumn{2}{|c|}{ Análise univariada } & \multicolumn{2}{|c|}{ Análise multivariada } \\
\hline & RP (IC95\%) & Valor de $p$ * & RP (IC95\%) & Valor de $p$ * \\
\hline Sexo & & 0,001 & & $<0,001$ \\
\hline Masculino & 1,00 & & 1,00 & \\
\hline Feminino & $0,89(0,84-0,95)$ & & $0,89(0,83-0,94)$ & \\
\hline Faixa etária (anos) & & 0,118 & & 0,064 \\
\hline $60-69$ & 1,00 & & 1,00 & \\
\hline $70-79$ & $1,05(0,98-1,11)$ & & $1,05(0,99-1,12)$ & \\
\hline 80 ou mais & $0,97(0,97-1,18)$ & & $1,09(0,99-1,21)$ & \\
\hline Cor da pele (referida) & & 0,515 & & $\star \star$ \\
\hline Branca & 1,00 & & $\star \star$ & \\
\hline Parda e preta & $0,97(0,88-1,07)$ & & ** & \\
\hline Escolaridade (anos) & & 0,692 & & $\star \star$ \\
\hline 12 ou mais & 1,00 & & $\star \star$ & \\
\hline $9-11$ & $1,04(0,94-1,14)$ & & $\star \star$ & \\
\hline $5-8$ & $1,01(0,92-1,11)$ & & $\star \star$ & \\
\hline $0-4$ & $0,99(0,92-1,07)$ & & $\star \star$ & \\
\hline Renda familiar per capita & & 0,835 & & $\star \star$ \\
\hline 4ㅇ quartil (maior) & 1,00 & & $\star \star$ & \\
\hline 3o quartil & $0,99(0,91-1,08)$ & & ** & \\
\hline 2o quartil & $1,03(0,94-1,13)$ & & $\star \star$ & \\
\hline 1ㅇ quartil & $0,99(0,89-1,12)$ & & ** & \\
\hline Tabagismo & & 0,513 & & $\star \star$ \\
\hline Nunca fumou & 1,00 & & $\star \star$ & \\
\hline Fumou e parou & $1,11(0,94-1,32)$ & & $\star \star$ & \\
\hline Fumante atual & $1,03(0,91-1,25)$ & & $\star \star$ & \\
\hline Consumo de bebida alcoólica & & 0,669 & & $\star \star$ \\
\hline Nunca & 1,00 & & $\star \star$ & \\
\hline Moderado & $1,04(0,92-1,18)$ & & ** & \\
\hline Alto & $1,03(0,91-1,17)$ & & $\star \star$ & \\
\hline Atividade física de lazer/deslocamento & & 0,304 & & $\star \star$ \\
\hline Fisicamente ativos & 1,00 & & ** & \\
\hline Insuficientemente ativos & $1,04(0,96-1,14)$ & & $\star \star$ & \\
\hline Dependência funcional & & 0,018 & & 0,030 \\
\hline Ausência de dependência & 1,00 & & 1,00 & \\
\hline Dependência leve & $1,09(0,98-1,22)$ & & $1,08(0,97-1,20)$ & \\
\hline Dependência moderada/grave & $1,15(1,02-1,09)$ & & $1,13(1,01-1,27)$ & \\
\hline IMC (peso/altura2) & & 0,678 & & $\star \star$ \\
\hline Peso adequado & 1,00 & & $\star \star$ & \\
\hline Baixo peso & $0,94(0,79-1,12)$ & & ** & \\
\hline Sobrepeso/Obesidade & $1,01(0,94-1,10)$ & & ** & \\
\hline Percepção em saúde & & $<0,001$ & & 0,004 \\
\hline Muito boa/Boa & 1,00 & & 1,00 & \\
\hline Regular & $1,09(1,01-1,17)$ & & $1,06(0,99-1,14)$ & \\
\hline Ruim/Muito ruim & $1,17(1,09-1,26)$ & & $1,13(1,04-1,22)$ & \\
\hline
\end{tabular}

(continua) 
Tabela 5 (continuação)

\begin{tabular}{|c|c|c|c|c|}
\hline \multirow[t]{2}{*}{ Variáveis } & \multicolumn{2}{|c|}{ Análise univariada } & \multicolumn{2}{|c|}{ Análise multivariada } \\
\hline & RP (IC 95\%) & Valor de $p$ * & RP (IC 95\%) & Valor de $p$ * \\
\hline Plano de saúde & & 0,655 & & $\star *$ \\
\hline Sim & 1,00 & & $\star \star$ & \\
\hline Não & $0,98(0,91-1,06)$ & & $\star \star$ & \\
\hline Consulta médica recente & & 0,003 & & 0,004 \\
\hline Não & 1,00 & & 1,00 & \\
\hline Sim & $1,14(1,05-1,25)$ & & $1,15(1,05-1,26)$ & \\
\hline
\end{tabular}

IC95\%: intervalo de 95\% de confiança; IMC: índice de massa corporal; RP: razão de prevalência.

* Valor de p corresponde ao teste de Wald da Regressão de Poisson;

** Variável excluída do modelo múltiplo por apresentar valor de $\mathrm{p}>0,05$.

pela grande cobertura da Estratégia Saúde da Família - ESF $(77,6 \%)^{32}$ e corroborado pelo fato de que $71,8 \%$ dos entrevistados tiveram consulta médica recente. Tal exposição aos serviços de saúde aumenta a probabilidade de diagnóstico da pressão arterial elevada, influenciando na prevalência do desfecho observada no presente trabalho 7,26,33.

A associação positiva da pressão arterial elevada com a faixa etária é concordante com a literatura 4,13 , ainda que no presente estudo esta relação não tenha sido linear 4 . É esperado que sua prevalência aumente com a idade, uma vez que alterações próprias do envelhecimento, como o enrijecimento de grandes artérias, tornam os idosos mais propensos ao desenvolvimento de HAS 28,34. Observou-se ainda que idosos com sobrepeso ou obesidade também apresentaram maior prevalência de pressão arterial elevada, fato que pode ser explicado por alterações fisiológicas como ativação do sistema nervoso simpático e renina-angiotensina-aldosterona, resistência à insulina e disfunção renal e endotelial 5. A relação entre excesso de gordura corporal e elevação da pressão arterial é relatada em inúmeras pesquisas, independentemente do indicador antropométrico considerado, sendo um dos principais fatores de risco para a HAS 35,36 . Neter et al. 37 , em uma metanálise, demonstraram haver redução média da pressão arterial de 1,05mmHg na sistólica e 0,92 mmHg na diastólica para cada diminuição de $1 \mathrm{~kg}$ de peso corporal, independentemente de idade, sexo ou raça.

Em relação à dependência funcional, maior prevalência de pressão arterial elevada foi associada a idosos com dependência funcional de moderada a grave, achado que está de acordo com outros estudos 23 , sendo esta a condição crônica que mais frequentemente influencia a capacidade funcional de idosos 6 . Além disso, os idosos constituem um importante grupo de risco para os eventos catastróficos e muitas vezes incapacitantes relacionados à HAS, tais como doença arterial coronariana, doença cerebrovascular, doença vascular periférica, insuficiência cardíaca, doença renal terminal e outras 5,26,28,38. $\mathrm{O}$ acidente vascular encefálico, que tem a hipertensão arterial sistêmica como o principal fator de risco modificável 39 , é uma das principais causas de dependência funcional nessa faixa etária, e tem o potencial de levar a complicações como incontinência urinária, disfagia, depressão e dor crônica 28.

Na presente pesquisa, verificou-se expressiva proporção de idosos que não conheciam sua condição de pressão arterial elevada (22,5\%). Outros estudos também indicaram elevada frequência de desconhecimento da condição clínica entre idosos, nos quais aproximadamente um em cada quatro hipertensos reportou não saber o diagnóstico da sua condição 7,8, alertando-se para a importância do acesso a serviços de saúde e da informação prestada ao idoso para o conhecimento da pressão arterial elevada 7 .

Constatou-se que houve maior prevalência de conhecimento da pressão arterial elevada entre as mulheres. Esse achado é consistente com outros estudos, que demonstram que as mulheres têm maior preocupação com sua saúde, apresentando maior tendência ao autocuidado e ao atendimento médico 7 . O conhecimento também foi mais frequente entre idosos de faixa etária mais avançada, sendo $25 \%$ maior entre aqueles com 80 anos ou mais, resultado este oposto ao verificado em outras pesquisas que não encontraram diferenças no conhecimento entre as faixas etárias 7 ou demonstraram maiores taxas de desconhecimento entre os mais idosos 40 .

No que diz respeito ao índice de massa corporal, o conhecimento da condição de pressão 
arterial elevada foi mais associado àqueles com índices mais altos, semelhante ao estudo de Nascente et al. 13, que encontraram associação entre indicadores antropométricos de gordura corporal e abdominal e hipertensão arterial referida em idosos, mas diferente de outro estudo 40 que apontou para indivíduos obesos como alvos almejados para campanhas de conscientização e atenção ambulatorial.

Em relação à percepção de saúde, observou-se que idosos com pior percepção de saúde conheciam mais sua condição, dados corroborados por outros estudos 7,8. A associação com pior percepção em saúde pode ser originada do fato de que o conhecimento da patologia foi um dos fatores que os respondentes utilizaram para classificar sua saúde como ruim ou regular 8 .

Idosos que se submeteram à consulta médica recente tiveram maior conhecimento da pressão arterial elevada. É de se esperar que o uso de serviços de saúde proporcione a chance de conhecimento da pressão arterial elevada, uma vez que aumenta a probabilidade de diagnóstico desta condição por profissional de saúde 7 , frequentemente assintomática. Assim, o uso de serviços de saúde pode aumentar o conhecimento, e este afeta diretamente a chance de tratamento e controle da pressão arterial elevada 7,33, sendo importante ressaltar que o não reconhecimento do diagnóstico pode ter grande repercussão negativa na adesão aos programas e tratamentos relativos à pressão arterial elevada 16 .

Neste estudo, dos 77,5\% de idosos que sabiam ter a doença, 20,9\% não estavam em tratamento, dado semelhante a outros trabalhos brasileiros $26,40,41$. O porcentual de hipertensos em tratamento varia amplamente em todo o mundo, desde $66,0 \%$ em Barbados até valores muito baixos, de apenas 10,7\% no México 26,42. A prevalência desse desfecho no Brasil foi significativamente superior em comparação a outros trinta países selecionados, especialmente em municípios com vasta cobertura da ESF, mostrando que esforços governamentais e de profissionais de saúde são fundamentais para se alcançar metas aceitáveis de tratamento e controle 4 . Dentre os fatores demográficos associados ao tratamento, observou-se que, apesar de estarem mais conscientes de sua condição, as mulheres tratam menos a pressão arterial elevada que os homens, divergindo de estudos nacionais e de outros países 26,41. Esse dado é inconsistente com o comportamento feminino ante outros indicadores de saúde, como dietas saudáveis, consumo e álcool e fumo ${ }^{41}$, uma vez que apresentam maior preocupação com a saúde e maior tendência ao autocuidado 7 .

Também verificou-se que idosos com dependência funcional apresentaram maior prevalên- cia de tratamento da pressão arterial elevada. A participação da família no cuidado dos idosos dependentes ajuda na adesão terapêutica, sendo relevante na aquisição de hábitos e mudanças no estilo de vida ${ }^{10}$, uma vez que o comprometimento da capacidade funcional inclui a dificuldade ou necessidade de auxílio para se tomar remédios na posologia correta 23 . O simples controle de doenças crônicas pode melhorar as atividades e, por conseguinte, promover o bem-estar desses indivíduos 6 .

A autopercepção em saúde ruim ou muito ruim demonstrou-se associada à maior prevalência de tratamento anti-hipertensivo neste estudo. A associação pode ser originada do fato de que o diagnóstico da doença foi um dos fatores que os idosos utilizaram para pior classificar sua saúde ${ }^{8}$, uma vez que o tratamento só foi avaliado entre os idosos que conheciam sua condição. Por outro lado, o fato de usar medicamentos por toda a vida, para tratamento de uma doença crônica e em geral assintomática 26 , frequentemente em uma associação de drogas, com esquemas difíceis e muitos efeitos indesejáveis $10,16,40$, pode influenciar a percepção de saúde dos idosos.

Ainda, observou-se que a prevalência do tratamento foi maior entre os idosos que se submeteram à consulta médica nos últimos 3 meses. Essa evidência corrobora relatos da literatura que afirmam que quanto melhor o acesso aos serviços de saúde maior o conhecimento da condição de pressão arterial elevada, que depende de diagnóstico clínico e, consequentemente, maiores deveriam ser as taxas de indivíduos em tratamento, maior a sua aderência e controle efetivo da pressão arterial 26.

O desenho de corte transversal deste estudo implica limitação na interpretação dos resultados, uma vez que não permite o estabelecimento de relações causais e nem temporais, sendo impossível definir se os fatores identificados como associados à prevalência, conhecimento e tratamento da pressão arterial elevada são consequentes da doença ou antecederam a sua ocorrência. No entanto, algumas associações analisadas e resultados encontrados parecem ser apropriados e compatíveis com registros de estudos coorte consultados 7,41. Quanto aos aspectos positivos, destaca-se a elevada taxa de resposta do presente trabalho e a moderada/alta taxa de concordância (valores de kappa entre 0,6 e 0,9 ) das variáveis inseridas no controle de qualidade.

Ainda que as políticas públicas em saúde devam ser universais, atenção especial deve ser direcionada aos subgrupos mais vulneráveis, tanto para ações de prevenção, diagnóstico e controle da hipertensão quanto para as ações de 
promoção à saúde 36 . Sendo assim, uma vez que se tem como ponto de partida a ilustração local do número de indivíduos acometidos, além de fatores associados à prevalência, conhecimento e tratamento da pressão arterial elevada, pode- se constituir como prioridade de saúde pública o direcionamento de esforços para a prevenção da hipertensão, o diagnóstico precoce de pressão arterial elevada e a garantia de tratamento aos diagnosticados positivamente.

\section{Resumen}

El objetivo fue estimar la prevalencia e investigar los factores asociados a la presión arterial elevada, su conocimiento y tratamiento en ancianos de Florianópolis, Santa Catarina, Brasil. Se trata de un estudio transversal de base poblacional y muestreo complejo. La presión arterial elevada fue definida por la medida del nivel de presión sanguínea el uso de antihipertensivos, el diagnóstico previo. La asociación de los desenlaces con las variables independientes fue verificada por la regresión de Poisson. Se entrevistaron a 1.705 ancianos. De ellos, un 84,6\% presentaron presión arterial elevada, un 77,5\% eran conscientes de la enfermedad y un 79,1\% la trataban. La prevalencia se asoció a la dependencia funcional y edad e índice de masa corporal (IMC) elevados. Ancianos del sexo femenino, edad e IMC elevados, peor percepción en salud y consulta médica reciente, eran más conscientes de la enfermedad. El tratamiento se asoció al sexo masculino, dependencia funcional, peor percepción en salud y consulta médica reciente. Pese a que políticas públicas en salud deben ser universales, es necesario considerar la desigual distribución de la presión arterial elevada en la población, dirigiéndose acciones de prevención, diagnóstico y garantía de tratamiento.

Hipertensión; Salud del Anciano; Prevalencia

\section{Colaboradores}

L. C. Zattar contribuiu substancialmente para a concepção e planejamento do artigo, para a análise e interpretação dos dados, para a elaboração do artigo e revisão crítica do conteúdo, além de participar da aprovação da versão final. A. F. Boing participou da concepção da análise dos dados, interpretação dos resultados e revisão crítica do texto. M. W. C. Giehl contribuiu substancialmente para a concepção e planejamento do artigo, bem como da análise e interpretação dos dados, contribuiu significativamente na elaboração do artigo e na revisão crítica do conteúdo, e participou da aprovação da versão final. E. d'Orsi participou da concepção do estudo, coleta de dados, análise dos dados, interpretação dos resultados e revisão crítica do texto. 


\section{Referências}

1. Chobanian A, Bakris G, Black H, Cushman W, Green L, Izzo JJ. The Seventh Report of the Joint National Committee on Prevention, Detection, Evaluation, and Treatment of High Blood Pressure: the JNC 7 report. JAMA 2003; 289:2560-72.

2. Lawes CM, Vander Hoorn S, Rodgers A; International Society of Hypertension. Global burden of blood-pressure-related disease, 2001. Lancet 2008; 371:1513-8.

3. Kearney PM, Whelton M, Reynolds K, Muntner P, Whelton PK, He J. Global burden of hypertension: analysis of worldwide data. Lancet 2005; 365:217-23.

4. Sociedade Brasileira de Cardiologia/Sociedade Brasileira de Hipertensão/Sociedade Brasileira de Nefrologia. VI Diretrizes Brasileiras de Hipertensão. Arq Bras Cardiol 2010; 95(1 Suppl 1):1-51.

5. Zaitune MPA, Barros MBA, César CLG, Carandina L, Goldbaum M. Hipertensão arterial em idosos: prevalência, fatores associados e práticas de controle no Município de Campinas, São Paulo, Brasil. Cad Saúde Pública 2006; 22:285-94.

6. Alves LC, Leimann BCQ, Vasconcelos MEL, Carvalho MS, Vasconcelos AGG, Fonseca TCO, et al. A influência das doenças crônicas na capacidade funcional dos idosos do Município de São Paulo, Brasil. Cad Saúde Pública 2007; 23:1924-30.

7. Firmo JOA, Uchôa E, Lima-Costa MF. Projeto Bambuí: fatores associados ao conhecimento da condição de hipertenso entre idosos. Cad Saúde Pública 2004; 20:512-21.

8. Méndez-Chacón E, Santamaría-Ulloa C, RoseroBixby L. Factors associated with hypertension prevalence, unawareness and treatment among Costa Rican elderly. BMC Public Health 2008; 8:275

9. Souza ARAD, Costa A, Diogo N, Mocheti LN, Stevanato PR, Ovando LA. Um estudo sobre hipertensão arterial sistêmica na cidade de Campo Grande, MS. Arq Bras Cardiol 2007; 88:441-6.

10. Pierin AMG, Gusmão JL, Carvalho LVB. A falta de adesão ao tratamento como fator de risco para hipertensão arterial. Rev Bras Hipertens 2004; 7:100-3.

11. Ribeiro HF, Plavnik FL, Kohlmann NEB. Aderência terapêutica. Rev Bras Hipertens 2010; 13:161-5.

12. Passos VMA, Assis TD, Barreto SM. Hipertensão arterial no Brasil: estimativa de prevalência a partir de estudos de base populacional. Epidemiol Serv Saúde 2006; 15:35-45.

13. Nascente FMN, Jardim PCBV, Peixoto MRG, Monego ET, Moreira HG, Vitorino PVO, et al. Hipertensão arterial e sua correlação com alguns fatores de risco em cidade de pequeno porte. Arq Bras Cardiol 2010; 95:502-9.

14. Pinto SL, Silva RCR, Priore SE, Assis AMO, Pinto EJ. Prevalência de pré-hipertensão e de hipertensão arterial e avaliação de fatores associados em crianças e adolescentes de escolas públicas de Salvador, Bahia, Brasil. Cad Saúde Pública 2011; 27:1065-76.

15. Lebrão ML, Laurenti R. Saúde, bem-estar e envelhecimento: o estudo SABE no Município de São Paulo. Rev Bras Epidemiol 2005; 8:127-41.

16. Firmo JOA, Lima-Costa MF, Uchôa E. Projeto Bambuí: maneiras de pensar e agir de idosos hipertensos. Cad Saúde Pública 2004; 20:1029-40.
17. Instituto Brasileiro de Geografia e Estatística. Estimativas da população para 1o de julho de 2009. Estimativas populacionais para TCU. http://www. ibge.gov.br/home/estatistica/populacao/estimati va2009/POP_DOU.pdf (acessado em 15/Mai/2010).

18. Programa das Nações Unidas para o Desenvolvimento. Atlas do Desenvolvimento Humano no Brasil, 2003. Brasília: Programa das Nações Unidas para o Desenvolvimento; 2003.

19. Hertz RP, Unger AN, Cornell JA, Saunders E. Racial disparities in hypertension prevalence, awareness, and management. Archives of Internal Medicine 2005; 165:2098-104.

20. Aziz MM, Calvo MCM, d'Orsi E. Medicamentos prescritos aos idosos em uma capital do Sul do Brasil e a Relação Municipal de Medicamentos. Cad Saúde Pública 2012; 28:52-64.

21. Babor TF, Higgins-Biddle JC, Saunders JB, Monteiro MG. AUDIT - The Alcohol Use Disorders Identification Test: guidelines for use in primary care. $2^{\text {nd }} \mathrm{Ed}$. Geneva: World Health Organization; 2001.

22. Matsudo S, Araujo T, Matsudo V, Andrade D, Andrade E, Oliveira LC, et al. Questionário Internacional de Atividade Física (IPAQ): estudo de validade e reprodutibilidade no Brasil. Rev Bras Ativ Fís Saúde 2001; 6:8-15.

23. Rosa TEC, Benício MHD, Latorre MRDO, Ramos LR. Fatores determinantes da capacidade funcional entre idosos. Rev Saúde Pública 2003; 37:40-8.

24. American Academy of Family Physicians/American Dietetic Association/National Council on the Aging. Nutrition screening and intervention resources for healthcare professionals working with older adults. Nutrition Screening Initiative. Washington DC: American Dietetic Association; 2002.

25. Barros AJ, Hirakata VN. Alternatives for logistic regression in cross-sectional studies: an empirical comparison of models that directly estimate the prevalence ratio. BMC Med Res Methodol 2003; 3:21.

26. Pereira MR, Coutinho MSSA, Freitas PF, d'Orsi E, Bernardi A, Hass R. Prevalência, conhecimento, tratamento e controle de hipertensão arterial sistêmica na população adulta urbana de Tubarão, Santa Catarina, Brasil, em 2003. Cad Saúde Pública 2007; 23:2363-74.

27. Longo GZ, Neves J, Luciano VM, Peres MA. Prevalence of high blood pressure levels and associated factors among adults in Southern Brazil. Arq Bras Cardiol 2009; 93:360-6.

28. Miranda RD, Perrotti TC, Bellinazzi VR, Nóbrega TM, Cendoroglo MS, Toniolo Neto J. Hipertensão arterial no idoso: peculiaridades na fisiopatologia, no diagnóstico e no tratamento. Rev Bras Hipertens 2002; 9:293-300.

29. Triantafyllou A, Douma S, Petidis K, Doumas M, Panagopoulou E, Pyrpasopoulou A, et al. Prevalence, awareness, treatment and control of hypertension in an elderly population in Greece. Rural Remote Health 2010; 10:1225.

30. Primatesta P, Poulter NR. Hypertension management and control among English adults aged 65 years and older in 2000 and 2001. J Hypertens 2004; 22:1093-8. 
31. Secretaria de Vigilância em Saúde/Secretaria de Gestão Estratégica e Participativa, Ministério da Saúde. Vigitel Brasil 2011: vigilância de fatores de risco e proteção para doenças crônicas por inquérito telefônico. Brasília: Ministério da Saúde; 2012.

32. Sisson MC, Andrade SR, Giovanella L, Almeida PF, Fausto MCR, Souza CRP. Estratégia de Saúde da Família em Florianópolis: integração, coordenação e posição na rede assistencial. Saúde Soc 2011; 20:991-1004.

33. Burt VL, Cutler JA, Higgins M, Horan MJ, Labarthe $\mathrm{D}$, Whelton $\mathrm{P}$, et al. Trends in the prevalence, awareness, treatment, and control of hypertension in the adult US population. Hypertension 1995; 26:60-9.

34. Cardoso CEP, Torejane D, Ghiggi RF. Evidências no tratamento da hipertensão arterial em idosos. Arquivos Catarinenses de Medicina 2006; 35:85-91.

35. Munaretti DB, Barbosa AR, Marucci MFN, Lebrão ML. Hipertensão arterial referida e indicadores antropométricos de gordura em idosos. Rev Assoc Med Bras 2011; 57:25-30.

36. Cutler J, Sorlie PD, Wolz M, Thom T, Fields LE, Roccella EJ. Trends in hypertension prevalence, awareness, treatment, and control rates in United States adults between 1988-1994 and 1999-2004. Hypertension 2008; 52:818-27.

37. Neter JE, Stam BE, Kok FJ, Grobbee DE, Geleijnse JM. Influence of weight reduction on blood pressure: a meta-analysis of randomized controlled trials. Hypertension 2003; 42:878-84.
38. Neder MM, Borges AAN. Hipertensão arterial sistêmica no Brasil: o que avançamos no conhecimento de sua epidemiologia? Rev Bras Hipertens 2006; 13:126-33.

39. Aronow WS, Fleg JL, Pepine CJ, Artinian NT, Bakris G, Brown AS, et al. ACCF/AHA 2011 Expert Consensus Document on Hypertension in the Elderly: a report of the American College of Cardiology Foundation Task Force on Clinical Expert Consensus Documents. Circulation 2011; 123:2434-506.

40. Gus I, Harzheim E, Zaslavsky C, Medina C, Gus M. Prevalência, reconhecimento e controle da hipertensão arterial sistêmica no Estado do Rio Grande do Sul. Arq Bras Cardiol 2004; 83:424-8.

41. Firmo JOA, Barreto SM, Lima-costa MF. The Bambui Health and Aging Study (BHAS): factors associated with the treatment of hypertension in older adults in the community. Cad Saúde Pública 2003; 19:817-27.

42. Kearney PM, Megan W, Kristi R, Paul WK, He J. Worldwide prevalence of hypertension: a systematic review. J Hypertens 2004; 22:11-9.

Recebido em 19/Jun/2012

Versão final reapresentada em 31/Out/2012

Aprovado em 13/Nov/2012 